\title{
Eruptive Signatures in the Solar Atmosphere During the WHI Campaign (20 March-16 April 2008)
}

\author{
Alphonse C. Sterling $\dagger$ \\ Space Science Office, VP62, NASA Marshall Space Flight Center, \\ Huntsville, AL 35812 USA \\ email: alphonse.sterling@nasa.gov
}

\begin{abstract}
We examined EUV movies of the Sun during the period of the Whole Heliospheric Interval (WHI) campaign of 20 March-16 April 2008, searching for indications of eruptive events. Our data set was obtained from EIT on SOHO, using its $195 \AA$ filter, and from EUVI on the two STEREO satellites, using their $171 \AA$, $195 \AA$, $284 \AA$, and $304 \AA$ filters. Here we present a table showing results from our preliminary search.
\end{abstract}

Keywords. Sun: activity, Sun: corona, Sun: coronal mass ejections (CMEs), Sun: flares, Sun: UV radiation

\section{Introduction}

Positive identification of the source regions on the solar disk of solar eruptions is, in many cases, a difficult problem. Of course there are frequently cases where there are obvious features on the solar disk, such as solar flares or strong intensity dimming regions, that occur in tandem with the launch of a coronal mass ejection (CME). There are other cases however where CMEs occur with only very weak solar-disk signatures, or even no signature at all - see, for example, (Hudson et al. (1998), Webb et al. (1998), Robbrecht et al. (2009)). During the Whole Heliospheric Interval (WHI) campaign of $20 \mathrm{March}-$ 16 April 2008, several satellites and ground-based observatories performed coordinated observations of the Sun and heliosphere, and therefore this period is an appropriate one for revisiting the connection between CMEs and their solar source regions. We have initiated such an investigation, and here we summarize some early results of an initial survey. Our focus was to identify visually what appear to be eruptive events in the lower solar atmosphere during the WHI period. This initial investigation can serve as a basis for more detailed studies to follow. Future investigations will make comparisons between our list with lists of observed CME eruptions.

\section{Analysis Procedure}

We first examined movies from the EUV Imaging Telescope (EIT) on the SOHO spacecraft over the WHI period for indications of eruptions, usually in the form of intensity dimmings (e.g., Hudson et al. (1998)), filament eruptions, or transient brightenings coupled with rapid changes in the local coronal structure (cf. Canfield et al. (1999)). From EIT we used images in the $195 \AA$ filter, as it had the highest cadence during the period ( $\sim 12 \mathrm{~min})$. After composing an initial list of low-coronal eruptive-like signatures with

$\dagger$ Present address: JAXA/Institute of Space and Astronautical Science, Hinode Group, 3-1-1 Yoshinodai, Sagamihara, Kanagawa 229-8510, Japan 
EIT $195 \AA$ images, we supplemented the list using movies from the EUVI imagers on the STEREO spacecraft. EUVI has better spatial resolution than EIT $(\sim 1.6 " /$ pixel vs. $2.6^{\prime \prime} /$ pixel $)$, and higher time cadence $(\sim 3-6 \mathrm{~min}$ for EUVI). We examined EUVI images taken with their $171 \AA, 195 \AA, 284 \AA$, and $304 \AA$ filters.

\section{Results}

During the WHI period, four active regions (ARs) appeared on the solar disk: NOAO ARs 10987, 10988, and 10989 appeared early in the WHI period, and 10990 began developing at the end of the WHI period. Many of the events we identified as "eruptive looking" emanated from or near the ARs. Table 1 presents an overview of our results. Seven of the events (events $3,7,10,12,15,16$, and 17) originated from an active region, while three of the events (events 4, 6, and 14), while near the limb, almost certainly had no direct connection to an active region. The connection between the remaining events and active regions was marginal or uncertain, as indicated in the Table 1 notes.

\section{Discussion}

We identified nearly 20 features that appeared eruptive, but not necessarily all were "ejective," i.e., an eruption that produced an ejection that left the Sun (e.g., Moore et al. (2001)). Some eruptions do not produce ejections into the heliosphere, sometimes even when the eruptions are accompanied by large flares (e.g., Green et al. (2002), Wang \& Zhang (2007)), but instead the material is confined by strong overlying magnetic field. Such "confined eruptions" often will not produce a CME (e.g., Moore et al. (2001), Török \& Kliem (2005)), or could result in a weak secondary CME or outflow (Bemporad et al. (2005)). To identify ejective eruptions we look for persistent dimmings, an expelled prominence, etc. Confined eruptions generally produce relatively transient dimmings or brightenings, and sometimes a filament can be seen to have its upward movement thwarted (e.g., Ji et al. (2003)). In marginal cases however it can be difficult to determine whether an eruption is confined or ejective, and so for several events in Table 1 we indicate the connection is uncertain. AR 10989 erupted several times, and some of these were clearly ejective. AR 10987 probably produced at least one ejective eruption (event 11), but overall ARs 10987 and 10988 displayed fewer eruptive signatures than AR 10989, according to our visual survey results.

In several cases we initially missed events in movies taken with the EIT and EUVI $195 \AA$ filter, but could identify them readily in movies from a different EUVI filter. This could be an important consideration when attempting to identify disk sources of CMEs.

\section{Acknowledgements}

A.C.S. was supported by funding from NASA's Office of Space Science through the Solar Physics Supporting Research and Technology Program and the Sun-Earth Connection Guest Investigator Program. The author thanks D. Webb and H. Cremades for valuable discussions.

\section{References}

Bemporad, A., Sterling, A. C., Moore, R. L., \& Poletto, G. 2005, ApJ, 635, L189

Canfield, R. C., Hudson, H. S., \& McKenzie, D. E. 1999, Geophys. Res. Lett., 26, 627

Green, L. M., Matthews, S. A., van Driel-Gesztelyi, L., Harra, L. K., \& Culhane, J. L. 2002, Solar Physics, 205, 325 
Hudson, H. S., Lemen, J. R., St. Cyr, O. C., Sterling, A. C., \& Webb, D. F. 1998, Geophys. Res. Lett., 25, 2481

Ji, H., Wang, H., Schmahl, E. J., Moon, Y.-J., \& Jiang, Y. 2003, ApJ, 595, L135

Moore, R. L., Sterling, A. C., Hudson, H. S., \& Lemen, J. R. 2001, ApJ, 552, 833

Robbrecht, E., Patsourakos, S., \& Vourlidas, A. 2009, ApJ, 701, 283

Török, T., \& Kliem, B. 2005, ApJ, 630, L97

Wang, Y., \& Zhang, J. 2007, ApJ, 665, 1428

Webb, D. F., Cliver, E. W., Gopalswamy, N., Hudson, H. S., \& St. Cyr, O. C. 1998, Geophys. Res. Lett., 25, 2469

Table 1. "Eruptive-Looking" Coronal Events Over WHI Period: 20-Mar - 16 Apr 2008

\begin{tabular}{|c|c|c|c|c|c|}
\hline Event & $\begin{array}{c}\text { Date } \\
(\mathrm{M} / \mathrm{D} \mathrm{D})\end{array}$ & $\mathrm{UT}$ & $\begin{array}{c}\text { First } \\
\text { Instrument }\end{array}$ & Location & Comments: \\
\hline \multirow[t]{2}{*}{1} & \multirow[t]{2}{*}{$3 / 22$} & \multirow[t]{2}{*}{$11: 45$} & \multirow[t]{2}{*}{ EUVI-B 195} & \multirow[t]{2}{*}{ At $E \lim b$} & Weak coronal opening or ejection. \\
\hline & & & & & $\begin{array}{l}\text { Possible precursor to } 14: 05 \text { UT } \\
\text { event. }\end{array}$ \\
\hline 2 & $3 / 22$ & $14: 05$ & EUVI-B 195 & At $E \operatorname{limb}$ & $\begin{array}{l}\text { Coronal opening, and ejection. Be- } \\
\text { hind the limb for EIT. Probably } \\
\text { from AR } 10989 \text { during its early } \\
\text { stages. }\end{array}$ \\
\hline 3 & $3 / 25$ & $18: 34$ & EIT 195 & AR 10989 at E limb. & $\begin{array}{l}\text { Coronal opening and dimming, } \\
\text { bright transient flare. }\end{array}$ \\
\hline- & $3 / 26$ & - & EUVI-B 195 & - & $\begin{array}{l}\text { Extensive activity between ARs } \\
10987 \text { and } 10988 \text {, but no obvious } \\
\text { ejections. Maybe a failed eruption } \\
\text { visible in EUVI-B } 284 \text { from about } \\
16: 26 \text { U between those two ARs, } \\
\text { perhaps producing a weak ejection } \\
\text { (cf. Bemporad et al. }(2005) \text { ). }\end{array}$ \\
\hline 4 & $3 / 27$ & c $16: 06$ & EUVI-B 304 , etc. & $\mathrm{NE} \operatorname{limb}$ in STEREO B. & $\begin{array}{l}\text { Slowly-erupting prominence in } \\
304 A \text {; faint in other EUVI filters. } \\
\text { Probably ejective, but not certain. }\end{array}$ \\
\hline 5 & $3 / 27$ & $17: 26$ & EUVI-B 284 & S of AR 10988 & $\begin{array}{l}\text { Filament erupts, but probably not } \\
\text { ejective. Source region rooted adja- } \\
\text { cent to but not in the AR, but AR } \\
\text { magnetic field may have triggered } \\
\text { onset. }\end{array}$ \\
\hline 6 & $3 / 29$ & $07: 47$ onward. & EIT 195 & A long E limb. & $\begin{array}{l}\text { Large-scale flows seen in } 195 \AA \text { fil- } \\
\text { ters and EUVI-B } 171 \AA ; \text { contin- } \\
\text { ues until next day. Slowly-evolving } \\
\text { prominence in EUVI-B } 304 \AA ; \text { may } \\
\text { eject from Sun at } \gtrsim 11 \text { U. }\end{array}$ \\
\hline 7 & $3 / 30$ & $05: 22$ & EIT 195 & A R 10989 . & $\begin{array}{l}\text { Eruptive flare, removing corona } \\
\text { (dimming) to SE. Also well seen in } \\
\text { EUVI-B } 284 .\end{array}$ \\
\hline 8 & $3 / 30$ & $>7: 46$ & EUVI-B 304 & S of ARs $10988 \& 10989$. & $\begin{array}{l}\text { Large filament eruption, faint in } \\
\text { non-304 filters. From EUVI-A } 304 \\
\text { however it may not be ejective. }\end{array}$ \\
\hline 9 & $4 / 01$ & $04: 26$ & EUVI-B 284 & $\mathrm{~N}$ of $\mathrm{AR} 10989$ & $\begin{array}{l}\text { Eruption with source outside of AR, } \\
\text { but with remote connections to the } \\
\text { AR. Prominent in } 284 \AA \text { but weak in } \\
195 \AA \text {. May be confined. }\end{array}$ \\
\hline 10 & $4 / 05$ & $05: 34$ & EIT 195 & AR $10989 \mathrm{SE}$ side. & $\begin{array}{l}\text { Eruption with dimming. From } \\
\text { EUVI- } \mathrm{B} 284 \AA \text { there may be a par- } \\
\text { tial ejection, but uncertain; EUVI } \\
171 \AA \text { shows dimming that suggests } \\
\text { an ejection however. }\end{array}$ \\
\hline 11 & $4 / 05$ & $15: 35$ & E U V I- A & $\begin{array}{l}\text { W } \operatorname{limb}, \text { probably } \\
\text { from AR } 10987\end{array}$ & Coronal opening and dimming. \\
\hline 12 & $4 / 05$ & $19: 34$ & EIT 195 & AR 10989, SE side. & $\begin{array}{l}\text { Same location as event at } 05: 34 \text { UT } \\
\text { on same day. Filament eruption } \\
\text { clear in } 304 \AA ; \text { probably ejective, } \\
\text { but uncertain. }\end{array}$ \\
\hline 13 & $4 / 09$ & $09: 58$ & EIT 195 & W limb, probably from AR 10989 & $\begin{array}{l}\text { Filament and cavity clearly erupting } \\
\text { in EUVI-A. In EUVI-B } 284 \text { appears } \\
\text { as very fast ejection. }\end{array}$ \\
\hline 14 & $4 / 11$ & $16: 06-24: 00$ & EUVI-B 304 & $\mathrm{NE} \operatorname{limb}$ & $\begin{array}{l}\text { Very slow filament eruption; seen in } \\
304 \AA \text { but not noticed in other fil- } \\
\text { ters. Faint and behind the limb in } \\
\text { EIT } 195 \AA \text {. }\end{array}$ \\
\hline 15 & $4 / 16$ & $06: 46$ & EUVI-B 284 & AR 10990 . & $\begin{array}{l}\text { Eruption from AR; possibly ejective, } \\
\text { but uncertain. }\end{array}$ \\
\hline 16 & $4 / 16$ & $10: 06$ & EUVI-B 284 & AR 10990 . & $\begin{array}{l}\text { Relatively strong eruption from } \mathrm{AR} \text {, } \\
\text { likely ejective. }\end{array}$ \\
\hline 17 & $4 / 16$ & $18: 06$ & EUVI-B 284 & A R 10990 . & Eruption from AR, likely ejective. \\
\hline
\end{tabular}

Notes:

${ }^{1}$ Instrument in which feature was first noticed. 